\title{
Profiling wind and greenhouse gases by infrared-laser occultation: results from end-to-end simulations in windy air
}

\author{
A. Plach, V. Proschek, and G. Kirchengast \\ Wegener Center for Climate and Global Change (WEGC) and Institute for Geophysics, Astrophysics and \\ Meteorology/Institute of Physics (IGAM/IP), University of Graz, Graz, Austria \\ Correspondence to: A. Plach (andreas.plach@uni-graz.at)
}

Received: 31 October 2014 - Published in Atmos. Meas. Tech. Discuss.: 12 January 2015

Revised: 17 June 2015 - Accepted: 22 June 2015 - Published: 16 July 2015

\begin{abstract}
The new mission concept of microwave and infrared-laser occultation between low-Earth-orbit satellites (LMIO) is designed to provide accurate and long-term stable profiles of atmospheric thermodynamic variables, greenhouse gases (GHGs), and line-of-sight (1.o.s.) wind speed with focus on the upper troposphere and lower stratosphere (UTLS). While the unique quality of GHG retrievals enabled by LMIO over the UTLS has been recently demonstrated based on end-to-end simulations, the promise of 1.o.s. wind retrieval, and of joint GHG and wind retrieval, has not yet been analyzed in any realistic simulation setting. Here we use a newly developed 1.o.s. wind retrieval algorithm, which we embedded in an end-to-end simulation framework that also includes the retrieval of thermodynamic variables and GHGs, and analyze the performance of both stand-alone wind retrieval and joint wind and GHG retrieval. The wind algorithm utilizes LMIO laser signals placed on the inflection points at the wings of the highly symmetric $\mathrm{C}^{18} \mathrm{OO}$ absorption line near $4767 \mathrm{~cm}^{-1}$ and exploits transmission differences from a wind-induced Doppler shift. Based on realistic example cases for a diversity of atmospheric conditions, ranging from tropical to high-latitude winter, we find that the retrieved l.o.s. wind profiles are of high quality over the lower stratosphere under all conditions, i.e., unbiased and accurate to within about $2 \mathrm{~m} \mathrm{~s}^{-1}$ over about 15 to $35 \mathrm{~km}$. The wind accuracy degrades into the upper troposphere due to the decreasing signal-to-noise ratio of the wind-induced differential transmission signals. The GHG retrieval in windy air is not vulnerable to wind speed uncertainties up to about $10 \mathrm{~m} \mathrm{~s}^{-1}$ but is found to benefit in the case of higher speeds from the integrated wind retrieval that enables correction of wind-induced Doppler shift of GHG signals. Overall both
\end{abstract}

the 1.o.s. wind and GHG retrieval results are strongly encouraging towards further development and implementation of a LMIO mission.

\section{Introduction}

This study is based on the mission concept "ACCURATE climate benchmark profiling of greenhouse gases and thermodynamic variables and wind from space" (Kirchengast et al., 2010). The ACCURATE concept utilizes inter-satellite cross links between low-Earth-orbit (LEO) satellites and represents a synergistic combination of the LEO-LEO microwave occultation (LMO) and the LEO-LEO infraredlaser occultation (LIO) technique. LMO is used to derive thermodynamic state variables, like pressure $(p)$, temperature $(T)$ and humidity $(q)$. Performed simultaneously from the same receiver and transmitter platforms as the LMO, the LIO is utilized to retrieve greenhouse gas (GHG) trace species concentrations and line-of-sight (l.o.s.) wind speed. The combined LEO-LEO microwave and infrared-laser occultation method (LMIO) is already well investigated and has undergone a range of studies introducing the concept and analyzing its feasibility and performance (Schweitzer, 2010; Kirchengast and Schweitzer, 2011; Schweitzer et al., 2011a, b; Proschek et al., 2011, 2014). In mission pre-development context it received positive evaluation, encouraging further studies, as part of the Earth Explorer 8 mission call (Kirchengast et al., 2010) from the European Space Agency.

The LMIO method, introduced by Kirchengast and Schweitzer (2011), is a next-generation step in the development of the well-established and meanwhile widely used ra- 


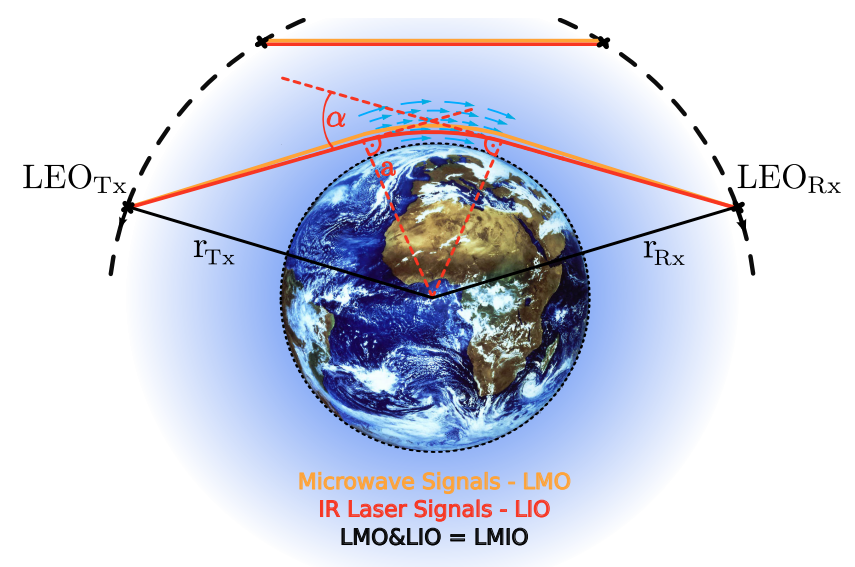

Figure 1. Schematic overview of the LMIO geometry and setup. The blue arrows symbolize the spherically symmetric 1.o.s. wind. Tx and $\mathrm{Rx}$ are the transmitter and receiver instruments on the LEO satellites. $r_{\mathrm{Tx}}$ and $r_{\mathrm{Rx}}$ are the radial distances from the transmitter and receiver to the center of curvature of the Earth. $\alpha$ denotes the bending angle of the IR signal, shown as a function of the impact parameter $a$.

dio occultation (RO) method which operates in the L-band region (Ware et al., 1996; Kursinski et al., 1997). In contrast to RO, LMIO utilizes a LEO satellite that actively transmits limb-sounding microwave (MW) and infrared-laser (IR) signals to a receiving LEO satellite. This setup is capable of providing independent, self-calibrated long-term stable measurements with high vertical resolution for climate monitoring and research (Kirchengast and Schweitzer, 2011).

The IR signals operate best under clear air conditions, which means no influence of clouds but of other atmospheric broadband effects like defocusing loss (Kursinski et al., 2000), Rayleigh scattering and aerosol extinction (Salby, 2012; Liou, 2002), and scintillations (Andrews and Philips, 2005; Gurvich et al., 2012). Schweitzer et al. (2011a) have analyzed these clear air influences in detail and Proschek et al. (2011) have studied the GHG retrieval performance under these conditions. Recently Proschek et al. (2014) have also carefully studied the influences of clouds and how retrievals successfully perform over broken cloudiness. In this study we avoid this part due to complexity and again use no clouds because we focus on the integrated retrieval of 1.o.s. wind speed, which has its core range of application in the lower stratosphere from about $15 \mathrm{~km}$ upwards above the typical tropospheric cloudiness. Only initial simplified studies regarding the 1.o.s. wind speed retrieval are available so far (Schweitzer, 2010; Kirchengast and Schweitzer, 2011), so this study is the first to analyze its performance in a realistic end-to-end simulation framework.

The LMIO observable altitude range focuses on the upper troposphere and stratosphere region $(\approx 5$ to $35 \mathrm{~km})$. The most important GHGs $\left(\mathrm{CO}_{2}, \mathrm{CH}_{4}, \mathrm{~N}_{2} \mathrm{O}, \mathrm{H}_{2} \mathrm{O}, \mathrm{O}_{3}, \mathrm{CO}\right.$; including key isotopes ${ }^{13} \mathrm{CO}_{2}, \mathrm{C}^{18} \mathrm{OO}, \mathrm{HDO}, \mathrm{H}_{2}^{18} \mathrm{O}$ ) can be re- trieved within an error range of 1 to $3 \%$ r.m.s., while the l.o.s. wind speed is expected to be derivable with good accuracy starting from about 15 up to $35 \mathrm{~km}$, with an expected monthly mean error range of $\pm 1 \mathrm{~m} \mathrm{~s}^{-1}$ (Kirchengast and Schweitzer, 2011). Both GHGs and 1.o.s. wind speed can be retrieved with a vertical resolution of about $1 \mathrm{~km}$.

For the retrieval of GHGs and l.o.s. wind speed the key step is the application of the differential transmission concept on an "on signal" (absorption signal) and "off signal" (reference signal) in order to essentially eliminate atmospheric broadband effects. In windy air, the IR signal frequencies are influenced by a l.o.s. wind-induced Doppler shift; as a result the GHG absorption signals, placed at the center of target absorption lines under zero wind, are somewhat affected and therefore the GHG volume mixing ratio (VMR) retrieval results are as well. The novelty of this study is the complementary implementation of a newly developed l.o.s. wind speed retrieval algorithm (Syndergaard and Kirchengast, 2013, 2015) into the overall LMIO algorithm developed at the Wegener Center. Within this framework we can quantitatively assess the l.o.s. wind speed retrieval performance as part of LMIO end-to-end simulations. Furthermore, we can analyze and demonstrate the benefit of a correction of the 1.o.s. wind-induced Doppler shift in the IR-laser signal frequencies used for GHG retrievals.

Figure 1 shows the LMIO geometry and illustrates MW and IR-laser signal ray paths in windy air conditions. The wind velocity vectors are indicated with blue arrows. The transmitter sends MW and IR-laser signals to a receiver while both satellites move in counter-rotating orbits; Kirchengast and Schweitzer (2011) explain the basics in detail. The signals undergo slightly different bending and their ray paths are indicated with different colors: orange for MW and red for IR. The IR-laser signals experience a frequency shift due to relative motion of transmitter and receiver, which practically corresponds, for wind speeds of several $10 \mathrm{~m} \mathrm{~s}^{-1}$, to a l.o.s. wind-induced Doppler shift $\Delta f / f$ in the range of $1 \times 10^{-7}$ at the IR-laser frequencies used for LMIO ( $f \approx 143 \mathrm{THz})$.

The wind retrieval is exploiting this Doppler shift to derive the 1.o.s. wind speed as conceptually introduced by Kirchengast and Schweitzer (2011). For the GHG-targeted absorption signals, which nominally sit at the center of selected GHG absorption lines, this Doppler frequency shift results in the observation of a slightly increased transmission (weaker absorption), since the frequency is shifted off the line center where maximum absorption would occur.

Here we show the performance of the l.o.s. wind speed retrieval based on the new Abel transform, compared to a simplified previous approach by Schweitzer (2010) and Kirchengast and Schweitzer (2011), and the benefit for the GHG retrieval of correcting the wind-induced Doppler shift in the GHG channels.

We show the LMIO retrieval performance for six representative occultation events using a transmitter and a receiver satellite in near-polar orbits (inclination $\approx 80^{\circ}$ ) as baselined 


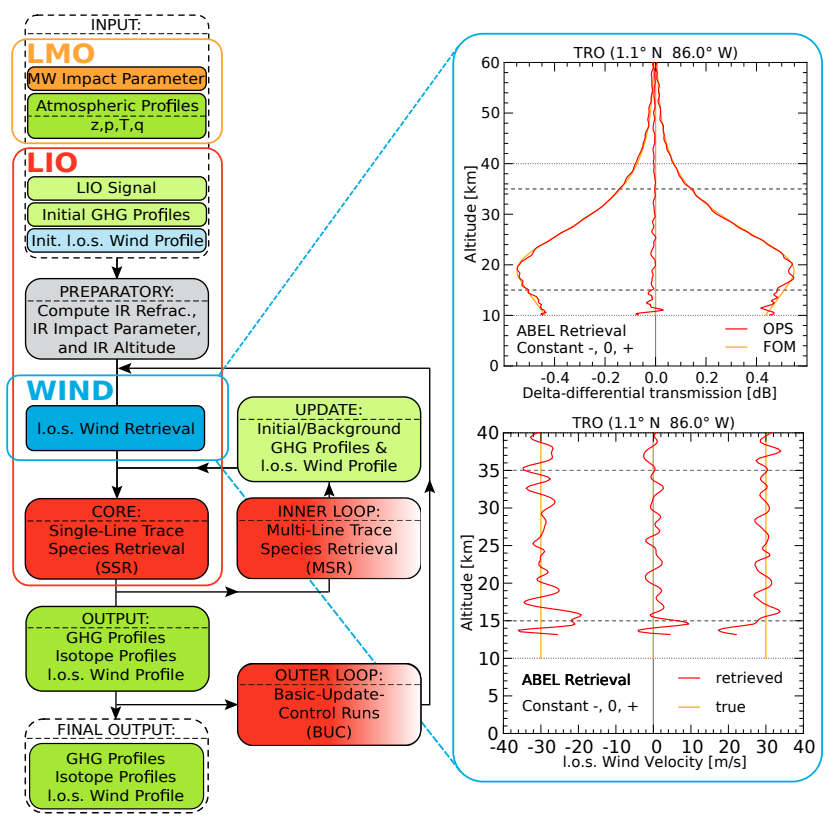

Figure 2. Schematic overview of the dynamical LIO wind and GHG retrieval algorithm, comprising the input parameters from LMO (orange framed box) and LIO (together with LMO in the dashed-black framed box) as well as the LIO retrieval's preparatory steps (gray box), wind retrieval step (blue box; exemplary delta-differential transmission and resulting wind profiles illustrated as "fly-out graphics" at the right-hand side where FOM denotes forward-modeled profiles and OPS (occultation processing system) denotes retrieved delta-differential transmission profiles), and core algorithm (SSR; red box). The GHG retrieval inner loop (MSR; red gradient box), computing and updating (light-green box) the GHGs in a pre-defined appropriate order, and the outer loop (basic-update-control runs; lower red gradient box), leading at the end to the final output (bottom green box), are sketched as well. Figure updated from Proschek et al. (2011).

by Kirchengast and Schweitzer (2011). Further details on the simulation setup are given in Sect. 3.

After this introduction the paper is structured as follows. In Sect. 2 we discuss the new l.o.s. wind retrieval algorithm and its integrated implementation within the so-called multispecies retrieval (MSR) part of the LIO retrieval algorithm in the eXtended End-to-End Generic Occultation Performance Simulation and Processing System (xEGOPS). We also discuss the advancement of the GHG retrieval algorithm to account for wind-induced Doppler shift. In Sect. 3 the setup for the end-to-end simulations is presented and in Sect. 4 the results of the performance analysis both for the new l.o.s. wind retrieval and for the GHG retrieval in windy air are presented. Section 5 finally provides the summary and conclusions.

\section{Integrating wind profiling into the LIO algorithm}

The first detailed description of the LMIO retrieval algorithm in clear air (defocusing loss, Rayleigh scattering, and aerosol extinction) was provided by Proschek et al. (2011). After that the algorithm was updated to add the capability of a GHG retrieval in cloudy air conditions by Proschek et al. (2014). The integration of a proper l.o.s. wind speed retrieval is the next crucial step in the LMIO development taken by this study. Based on the assumption in the wind algorithm that the wind velocity in the occultation plane near the tangent point location is layered in a spherically symmetric way, the retrieval utilizes an Abel transform to calculate the l.o.s. wind speed profile from measured transmission profile data. By applying an Abel transform we are able to accurately retrieve wind profiles that vary rather strongly with altitude, i.e., we can account also for strong vertical wind shears.

The derivation of an adequate Abel transform that provides the relationship between l.o.s. wind speed and the observed (delta-differential) transmission of the two thoroughly chosen wind channels (plus a reference channel) was done in detail by Syndergaard and Kirchengast $(2013,2015)$. Therefore only the resulting key equations are summarized here. The focus of this section is the discussion of the new wind algorithm and its implementation within the MSR part of the xEGOPS simulation software.

The existing GHG retrieval algorithm from LIO is the backbone of the new wind retrieval; a detailed description can be found in Proschek et al. (2011). The dynamic algorithm flow, with the wind profiling inserted, is presented in Fig. 2. The following description can be seen as an update to the algorithm descriptions in Proschek et al. $(2011,2014)$. Only additional key steps within the overall algorithm and changes in relation to the descriptions in these two publications will be discussed here. The so-called simple 1.o.s. wind retrieval, which is used for comparison later on in this paper, uses the assumption of a constant l.o.s. wind speed along the entire occultation ray path. This simple retrieval was introduced and described by Schweitzer (2010) and Kirchengast and Schweitzer (2011) and will not be further discussed here.

\subsection{The new l.o.s. wind profiling algorithm}

All absorption and reference channels used within the GHG retrieval are vulnerable to the l.o.s. wind-induced Doppler shift. There are three major contributors to the IR-laser signal's Doppler shift: motion of the transmitter and the receiver, Earth's rotation, and the l.o.s. wind speed prevailing in the atmosphere in the occultation plane around the tangent point location. For a frequency $f \approx 143 \mathrm{THz}\left(\mathrm{C}^{18} \mathrm{OO}\right.$ channels near $4767 \mathrm{~cm}^{-1}$ ) this corresponds to Doppler shifts $\Delta f / f$ of near $2 \times 10^{-5}, 1 \times 10^{-6}$, and $1 \times 10^{-7}$, respectively (Syndergaard and Kirchengast, 2013, 2015). The influence of the two larger shifts can be predicted and accounted for accurately in the instrument design, leaving uncertainties smaller 
Table 1. Infrared-laser absorption and reference channels used for the study (wavenumber data based on HITRAN 2008).

\begin{tabular}{cccc}
\hline $\begin{array}{c}\text { Target } \\
\text { species }\end{array}$ & $\begin{array}{c}\text { Absorption } \\
\text { wavenumber } \\
\mathrm{cm}^{-1}\end{array}$ & $\begin{array}{c}\text { Reference } \\
\text { wavenumber } \\
\mathrm{cm}^{-1}\end{array}$ & $\begin{array}{c}\text { (Abs. - Ref.)/Ref. } \\
\text { freq. spacing } \\
\%\end{array}$ \\
\hline $\mathrm{C}^{18} \mathrm{OO}-\mathrm{w} 1$ & 4767.037455 & 4770.15 & -0.0653 \\
$\mathrm{C}^{18} \mathrm{OO}-\mathrm{w} 2$ & 4767.045455 & 4770.15 & -0.0651 \\
${ }^{12} \mathrm{CO}_{2}$ & 4771.621441 & 4770.15 & +0.0308 \\
${ }^{13} \mathrm{CO}_{2}$ & 4723.414985 & 4731.03 & -0.1610 \\
$\mathrm{CH}_{4}$ & 4344.163500 & 4322.93 & +0.4912 \\
$\mathrm{H}_{2} \mathrm{O}-1$ & 4204.840290 & 4227.07 & -0.5259 \\
$\mathrm{H}_{2} \mathrm{O}-2$ & 4775.802970 & 4770.15 & +0.1185 \\
$\mathrm{H}_{2} \mathrm{O}-3$ & 4747.054840 & 4731.03 & +0.3387 \\
$\mathrm{H}_{2} \mathrm{O}-4$ & 4733.045010 & 4731.03 & +0.0426 \\
$\mathrm{O}_{3}$ & 4667.115600 & 4670.17 & -0.0655 \\
\hline
\end{tabular}

than $10^{-8}$ so that the 1.o.s. wind is well observable. In particular, these highly predictable kinematic Doppler shifts are foreseen to be compensated on the fly in an LMIO mission by adequate transmitter frequency design; the recent LMIO mission proposal by Kirchengast et al. (2010) addressed this need.

The remaining Doppler shift caused by the prevailing 1.o.s. wind speed, if not corrected for, adds a small bias to the retrieved GHG profiles in case of wind speeds exceeding $10 \mathrm{~m} \mathrm{~s}^{-1}$ (cf. Schweitzer et al., 2011a, Sect. 3.8.3 therein). Typical biases for wind speeds of several $10 \mathrm{~m} \mathrm{~s}^{-1}$ are of the magnitude of $1 \%$ in the VMR of trace species. However, it is this wind-induced Doppler shift that is exploited to derive 1.o.s. wind speed profiles from transmission profiles of the wind-sensitive channels near $4767 \mathrm{~cm}^{-1}$ at the highly symmetric $\mathrm{C}^{18} \mathrm{OO}$ absorption line. These Doppler shift estimates are then subsequently used to avoid the bias of the GHG profiles.

Generally it is possible with this method to retrieve l.o.s. wind speeds between about \pm 1 and $\pm 100 \mathrm{~m} \mathrm{~s}^{-1}$ (lower limit from signal-to-noise ratio, upper limit from width of absorption line), which covers the relevant wind velocities occurring in Earth's troposphere and stratosphere well (Salby, 2012).

The new l.o.s. wind speed retrieval algorithm starts with the transmission profiles at two wind-sensitive absorption channels $\left(v_{\mathrm{w} 1}, v_{\mathrm{w} 2}\right)$, located at the inflection points of the highly symmetric $\mathrm{C}^{18} \mathrm{OO}$ absorption line at $4767.041455 \mathrm{~cm}^{-1} \pm 0.0004 \mathrm{~cm}^{-1}$, and the corresponding reference channel at $4770.15 \mathrm{~cm}^{-1}$. Table 1 includes these frequencies. There are some preparatory steps necessary to provide these transmission profiles as a function of impact parameter ("IR impact parameter") and altitude ("IR altitude"), shown in Fig. 2 in a gray box (the impact parameter is the perpendicular distance between the ray path and the Earth's center of curvature in the occultation event plane; see Proschek et al., 2011, for details). These steps employ exist- ing algorithms, which are discussed in an overview style in Sect. 2.2 below.

The individual wind channel transmission profiles on the IR impact parameter grid and the corresponding reference channel transmission profile are used to derive the differential transmission profiles for the two wind channels, which are then smoothed using a third-order sliding polynomial filter with a width of about $1 \mathrm{~km}$. Finally, the so-called deltadifferential transmission is calculated following

$\Delta \mathcal{T}(a)=\mathcal{T}_{\mathrm{w} 1}(a)-\mathcal{T}_{\mathrm{w} 2}(a)$,

where $a$ denotes the impact parameter and where $\mathcal{T}_{\mathrm{w} 1}(a)$ and $\mathcal{T}_{\mathrm{w} 2}(a)$ denote the differential transmissions $\left(\mathcal{T}_{\text {abs }}-\mathcal{T}_{\text {ref }}\right)$, respectively, for the two wind channels $v_{w 1}$ and $v_{\mathrm{w} 2}$. Therein, $\mathcal{T}_{\text {abs }}$ is the transmission at the absorption channel (online) and $\mathcal{T}_{\text {ref }}$ the transmission at the reference channel (offline). An example for the delta-differential transmission $\Delta \mathcal{T}(a)$ behavior as a function of altitude can be seen in Fig. 2 in the upper right panel, both for constant $\pm 30 \mathrm{~m} \mathrm{~s}^{-1}$ wind and for zero wind influence.

The main equation for the l.o.s. wind speed retrieval via an Abel transform (as a function of the IR impact parameter $a$ ) is

$v(a) \approx \frac{\mathrm{c}}{\Delta \chi_{0}(a)}\left[\frac{1}{\pi} \frac{\mathrm{d}}{\mathrm{d} r} \int_{a}^{\infty} \frac{\Delta \tau(x) \mathrm{d} x}{\sqrt{x^{2}-a^{2}}}+\Delta k_{0}(a)\right]$,

with

$\Delta \tau(x)=-\frac{\Delta \mathcal{T}(a)}{10 \log (e)}$

and

$\Delta k_{0}(a)=k_{\mathrm{w} 1}(a)-k_{\mathrm{w} 2}(a)$,

$\Delta \chi_{0}(a)=\left.v_{\mathrm{w} 1} \frac{\mathrm{d} k_{\mathrm{w} 1}(a)}{\mathrm{d} v}\right|_{\nu_{\mathrm{w} 1}}-\left.v_{\mathrm{w} 2} \frac{\mathrm{d} k_{\mathrm{w} 2}(a)}{\mathrm{d} v}\right|_{\nu_{\mathrm{w} 2}}$.

Equations (2), (4), and (5) were derived in Syndergaard and Kirchengast $(2013,2015)$, which contain further details. The main input variables of Eq. (2) are the volume absorption coefficients at the two wind channels $k_{\mathrm{w} 1}(a)$ and $k_{\mathrm{w} 2}(a)$ (Eq. 4), their spectral derivatives $\left.\frac{\mathrm{d} k_{\mathrm{w} 1}(a)}{\mathrm{d} v}\right|_{\nu_{\mathrm{w} 1}}$ and $\left.\frac{\mathrm{d} k_{\mathrm{w} 2}(a)}{\mathrm{d} v}\right|_{\nu_{\mathrm{w} 2}}$ (Eq. 5), and the delta-differential optical depth $\Delta \tau(x)$ converted from the delta-differential transmission $\Delta \mathcal{T}(a)[\mathrm{dB}]$ (Eq. 3). Further variables are the speed of light, $c$, and the impact parameter, $a$.

Before the $\Delta \mathcal{T}(a)$ and the other key variables can be put into the Abel transform for calculating the 1.o.s. wind speed (Eq. 2), a few preparatory steps are necessary. The external reference forward model (RFM) (Edwards, 1996; Dudhia, 2008), a line-by-line radiative transfer model, is called four times to get the volume absorption coefficients, $k_{\mathrm{w} j \pm \mathrm{h}}(a) \pm \mathrm{a}$ small increment $h$, displaced from the two wind channels. These values are used to numerically compute a finite-difference estimate of the derivatives $\left.\frac{\mathrm{d} k}{\mathrm{~d} \nu}\right|_{v_{\mathrm{w} j}}$ and 

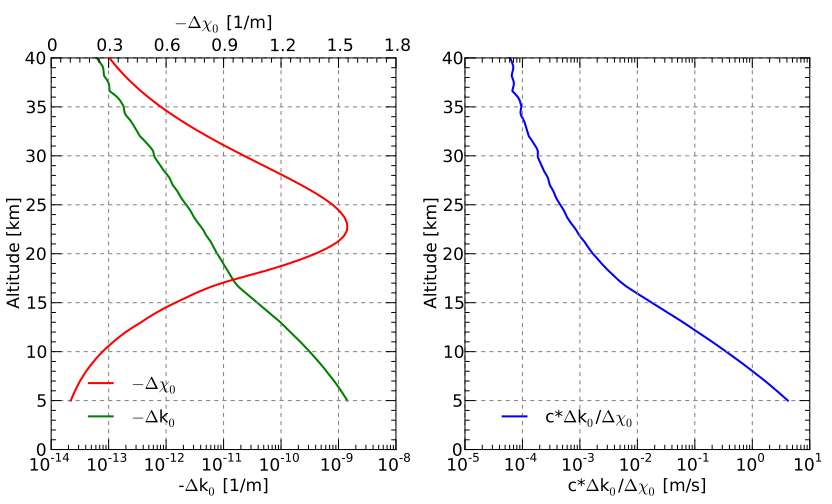

Figure 3. Left: profiles of two key input variables into the new Abel transform for the l.o.s. wind profiling. $\Delta k_{0}$ is a differential volume absorption coefficient between the wind channels and $\Delta \chi_{0}$ an associated differential absorption coefficient derivative. Right: profile of the " $k$ term", which appears as the small second term in the wind Abel transform (Eq. 2).

subsequently compute $\Delta \chi_{0}(a)$ (Eq. 5). In order to save computational time (by minimizing the number of RFM calls) the values of $k_{\mathrm{w} j}(a)$, needed for computing $\Delta k_{0}(a)$ (Eq. 4), are obtained by just taking the mean of the two adjacent values at $k_{\mathrm{w} j+\mathrm{h}}(a)$ and $k_{\mathrm{w} j-\mathrm{h}}(a)(j=1,2$ denoting the two wind channels). Sensitivity tests showed that these numerical estimates of the needed variables are very robust and accurate.

Figure 3 illustrates key ingredient variables of the Abel integral, Eq. (2). The left panel is designed to convey the magnitude and the altitude dependency of the two major inputs of Eq. (2), $\Delta k_{0}(a)$ and $\Delta \chi_{0}(a)$, in addition to $\Delta \tau(x)$. $\Delta k_{0}(a)$, the differential volume absorption coefficient between the wind channels (units $\mathrm{m}^{-1}$ ), shows an exponential dependency and values between about $-1 \times 10^{-9}$ and $-1 \times 10^{-13}$ over 5 to $40 \mathrm{~km} . \Delta \chi_{0}(a)$, the Doppler shift sensitivity of the volume absorption coefficient (units $\mathrm{m}^{-1}$ ), however, shows a clear maximum magnitude between 20 and $25 \mathrm{~km}$ and ranges between about -0.1 and -1.5 within 5 to $40 \mathrm{~km}$. The right panel of Fig. 3 shows the dependency of the second term in Eq. (2) (units $\mathrm{m} \mathrm{s}^{-1}$ ), the small "k term" that is added to the Abel integral term. This term again shows an essentially exponential dependency, ranging from about 5 to $5 \times 10^{-5}$ over 5 to $40 \mathrm{~km}$.

\subsection{Greenhouse gas retrieval algorithm under windy air conditions}

The GHG retrieval under windy air conditions is an update of the dynamic-loop part of the LMIO algorithm, as introduced above, named MSR loop, which was described in detail by Proschek et al. (2011). Figure 2 illustrates how the modification from wind retrieval insertion comes into the play, integrated with the retrieval of GHGs (and GHG isotopes). We focus on describing this add-on and refer for the basic description to Proschek et al. (2011).
The essential steps for the 1.o.s. wind/GHG retrievals happen in the LIO part (red frame). The main input variables of the LIO retrieval are the transmitter/receiver positions and the transmitted LIO signal intensities on the time grid shared with the LMO signals. Furthermore, initial GHG profiles (light green boxes) and an initial l.o.s. wind speed profile (light blue box) on the common altitude ( $z$ ) grid (shared with LMO parameters) are used as an LIO input.

The MW impact parameter in combination with the thermodynamic state variables $(p, T, q)$ are used to calculate the required refractive and geometric IR quantities as preparatory steps (gray box). These preparatory steps are necessary since the needed excess phase observations for deriving these quantities are only accessible in the MW domain and not from the IR intensity-only measurements (IR signal phase tracking would not be robust). The essential derived IR quantities are the IR impact parameter and the IR tangent point altitude. These IR parameters provide an altitude scale, consistent with the LMO data, for the LIO intensity profiles measured as a function of time. This special IR altitude grid estimation is necessary, because the MW and the IR occultation ray paths show (small) differences in bending that need to be carefully accounted for, especially in the upper troposphere where the humidity is increasing. After the preparatory steps, the new 1.o.s. wind speed retrieval component (blue box) is inserted, including the new algorithm after Syndergaard and Kirchengast $(2013,2015)$ as summarized in Sect. 2.1 above.

Next, the single-line trace species retrieval (SSR; red box) in Fig. 2 denotes the sequence of steps that are done to perform the retrieval of a single GHG species. The first key step for the present purpose is the derivation of differential transmission profiles from each pair of LIO intensity profiles (absorption and corresponding reference channel; see Table 1 for the target species absorption lines and frequencies used) and their allocation to the IR altitude grid. Then the differential transmission $\Delta \mathcal{T}\left(z_{i} ; v_{j}\right)$ and the modeled species transmission $\Delta \mathcal{T}_{\mathrm{bgr}}\left(z_{i} ; v_{j}\right)$ (transmission caused by foreign species; derived from an RFM call with the initial/background GHG profiles) are used to isolate the target species transmission $\Delta \mathcal{T}_{\text {tgt }}\left(z_{i} ; v_{j}\right)$ of the absorption channel. In this way the transmission purely due to an individual target GHG becomes known with only negligible influence of foreign species. A so-called absorptive Abel transform (Kursinski et al., 2002; Schweitzer et al., 2011a) is then used to derive the target species absorption coefficient on the IR altitude grid. Finally, the target species absorption coefficient and a modeled absorption cross section of the target species (via RFM based on initial/background target species profile and $p$ and $T$ profiles from LMO) are used to calculate the retrieved GHG VMR profile. This is the core algorithm. The results are iteratively improved due to subsequent wind and GHG updates, which are explained further down.

The MSR ("inner loop" red box in Fig. 2) provides a loop to perform a multiple species retrieval in a consecutive, thoroughly defined order over single-line species retrievals (see 
Table 1 in Proschek et al., 2011). This order ensures that the initial/background GHG profiles are updated so that the SSR can use an improved profile set for every new species retrieval within the MSR loop.

It is possible and useful to already perform the first l.o.s. wind speed retrieval using an initial Doppler shift estimate for the GHG correction of the differential transmission profiles of the two wind channels $\left(\Delta \mathcal{T}_{\mathrm{w} 1}, \Delta \mathcal{T}_{\mathrm{w} 2}\right)$. This Doppler shift estimate can be based on a co-located wind profile from an ECMWF short-range ( $24 \mathrm{~h}$ ) forecast field, which we will do in the end-to-end simulations presented in this paper. This approach would also be available for operational processing of real LMIO data; it already improves the retrieval at first step (while the same end result after iterations would be reached also for a start with zero wind, i.e., there is no actual a priori dependence). The wind retrieval itself is done as described in the preceding Sect. 2.1.

During the algorithm flow the initial l.o.s. wind speed profile is allocated to the background profile, which is updated after each retrieval with the retrieved l.o.s. wind speed profile between 14 and $45 \mathrm{~km}$, wherein the retrieval works well. At the margins of this altitude range there is a smooth transition (half-sine-weighted, $2 \mathrm{~km}$ width) to the initial l.o.s. wind speed profile from the ECMWF forecast field, completing the profile below and above for its use for Doppler shift correction in the GHG retrieval. The altitude range from about 15 to $45 \mathrm{~km}$ represents the domain where an accurate 1.o.s. wind speed retrieval within the scientific target observational requirements of the LMIO mission concept (Kirchengast et al., 2010) is found possible. The sign of the l.o.s. wind speed is set by the l.o.s. wind direction, which we defined as positive when the prevailing wind is blowing towards the receiver.

The wind correction in the GHG retrieval is implemented as follows. Within the MSR loop, the individual-channel frequency profiles $v_{j}$, originally filled with the nominal frequency $v_{0}$ of the absorption and reference channels, are adjusted by the Doppler shift induced by the l.o.s. wind speed, $v_{\text {l.o.s. }}$. This frequency profile adjustment, formulated on the altitude grid $z_{i}$, reads as follows:

$$
\begin{aligned}
& v_{\mathrm{Abs}, j}\left(z_{i}\right)=v_{\mathrm{Abs}, 0}\left(z_{i}\right)\left(1-\frac{v_{\text {l.o.s. }}\left(z_{i}\right)}{c}\right), \\
& \nu_{\text {Ref }, j}\left(z_{i}\right)=v_{\text {Ref }, 0}\left(z_{i}\right)\left(1-\frac{v_{\text {l.o.s. }}\left(z_{i}\right)}{c}\right),
\end{aligned}
$$

where $c$ is the vacuum speed of light and $j$ serves to index the absorption and reference channel pairs of single GHG species.

Furthermore, the Doppler shift correction is also used to improve the foreign GHG correction in the SSR algorithm (red box in Fig. 2). That is, the frequency shift information is used during the performance of the GHG background correction of the differential transmission profile (cf. Proschek et al., 2011, Sect. 3.4.2 therein), where the target species transmission now in windy air is calculated by

$\mathcal{T}_{\text {tgt }}\left(z_{i} ; v_{j}\right)=\Delta \mathcal{T}\left(z_{i} ; v_{j}\right)-\Delta \mathcal{T}_{\text {bgr }}\left(z_{i} ; v_{j}\right)$,

where

$\Delta \mathcal{T}\left(z_{i} ; v_{j}\right)=\mathcal{T}_{\mathrm{Abs}}\left(z_{i} ; v_{j}\right)-\mathcal{T}_{\operatorname{Ref}}\left(z_{i} ; v_{j}\right)$

and

$\Delta \mathcal{T}_{\text {bgr }}\left(z_{i} ; v_{j}\right)=\mathcal{T}_{\text {Abs,bgr }}\left(z_{i} ; v_{\text {Abs }, j}\right)-\mathcal{T}_{\text {Ref,bgr }}\left(z_{i} ; v_{\text {Ref }, j}\right)$.

Equations (8) to (10) are updated from Proschek et al. (2011) (Eqs. 13 to 15 therein). From $\Delta \mathcal{T}_{\text {tgt }}\left(z_{i} ; v_{j}\right)$ onwards the algorithm again follows the clear air algorithm as described in Proschek et al. (2011) (cf. Fig. 2 with Fig. 2 in Proschek et al., 2011).

The 1.o.s. wind-corrected frequencies are applied for each GHG species within the MSR loop so that the background GHG profiles are updated with retrieved GHG profiles that are already corrected for 1.o.s. wind-induced Doppler shift. After the inner loop is finished the first time, the basic run of the MSR loop is completed for all GHG species.

After this basic run, the so-called outer loop (basicupdate-control loop) is initiated. It takes the GHG and l.o.s. wind output profiles and feeds them again, as new background profiles, into the algorithm. In this way the 1.o.s. wind speed retrieval is further improved again during the update run, and subsequently the GHG retrieval is also improved during the update MSR loop. Another complete run, the control run that uses the outputs of the update run as input, finally ensures and controls the convergence of the l.o.s. wind speed and GHG results; its results nominally have negligible deviation from the results of the update run as demonstrated by Proschek et al. (2011).

\section{End-to-end simulation setup}

We performed LMIO end-to-end simulations with the Endto-End Generic Occultation Performance Simulation and Processing System (EGOPS) and eXtended EGOPS (xEGOPS) (Fritzer et al., 2010) using realistic atmospheric conditions for MW and IR-laser signals. The xEGOPS system is a development environment based on the operational EGOPS system (Fritzer et al., 2009) that performs the LMO end-toend simulations. xEGOPS adds the LIO end-to-end simulations using the EGOPS kernel library and is designed to enable the integration of such new algorithms under development.

In this section we discuss the setup of the simulations and the atmospheric thermodynamic, wind, and GHG conditions. The first step of the end-to-end simulations is the calculation of geometric orbit arcs of the transmitter and receiver satellites and of the geometric tangent point locations for every occultation event. These calculations were done with the mission analysis/planning sub-tool of the EGOPS/xEGOPS system. We used near-polar orbits with an inclination of $\approx 80^{\circ}$, 


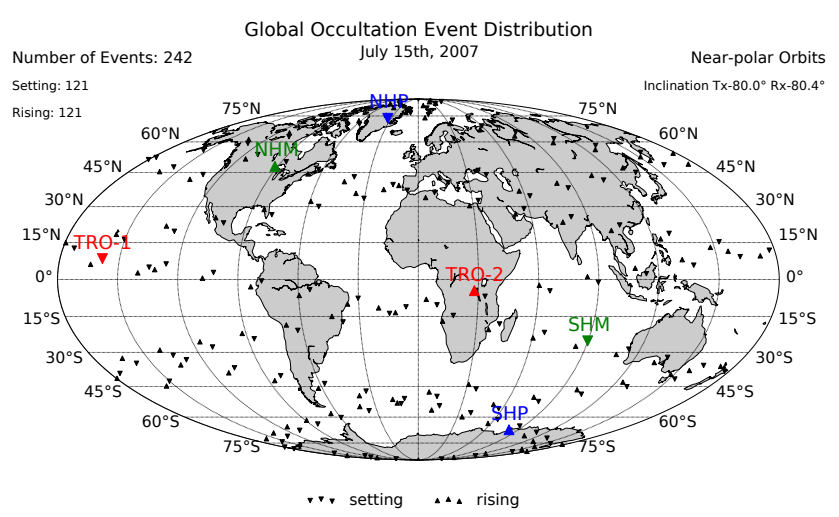

Figure 4. Global distribution of the occultation events on 15 July 2007 using near-polar $\left(i \approx 80^{\circ}\right)$ orbits. Rising events are indicated with upright triangles, while setting events are represented using reversed (upside-down) triangles. The six occultation event locations used for the study are colored in red, green, and blue according to their latitude band.

in line with the ACCURATE mission concept proposal of Kirchengast et al. (2010). Based on the occultation event simulations of a full day, six representative example events were selected to represent different atmospheric conditions. The global distribution of the occultation events is illustrated in Fig. 4.

The next step of simulation is the propagation of the MW and the IR-laser signals through the atmosphere, which was carried out with the forward modeling sub-tool of the EGOPS/xEGOPS system. We use an elliptical Earth figure model (World Geodetic System 1984; WGS84), spherical symmetry of the atmosphere at the occultation event locations, and a sampling rate of $10 \mathrm{~Hz}$. Both the MW and IR-laser signals undergo refraction, defocusing, and absorption loss (Kursinski et al., 2000). The IR-laser signals were treated to be additionally influenced by aerosol extinction (medium aerosol load), Rayleigh scattering, and scintillations (Schweitzer et al., 2011a).

We calculate the IR absorption with the RFM model, employing the High Resolution Transmission (HITRAN) 2008 (Rothman et al., 2009) database for the spectroscopic line parameters. We cross-checked the more recent HITRAN 2012 database (Rothman et al., 2013) and found that any changes are very small for our selected lines and would not impose any relevant change to the results. We therefore kept consistency with the recent Proschek et al. (2014) end-to-end simulation study that also used HITRAN 2008.

Table 1 summarizes the wavenumbers used for the absorption and reference channels plus the corresponding frequency spacing. The representative GHG concentrations for the simulations of the molecular absorption were taken from the Fast Atmospheric Signature Code model (FASCODE, 2008). This RFM/HITRAN/FASCODE subsystem is integrated in the forward modeling sub-tool of the EGOPS/xEGOPS sys- tem so that realistic IR absorption computations along ray propagation paths are seamlessly possible.

Regarding spectroscopic uncertainties, the HITRAN 2008 database used does provide indication of uncertainties of the spectroscopic parameters, with the line intensity and the airbroadened half-width being the main contributors (Harrison et al., 2011; Rothman et al., 1998, Appendix A2 therein). Harrison et al. (2011) studied the spectroscopic requirements for an ACCURATE-type mission concept and concluded that the current spectroscopic knowledge on the targeted absorption lines needs improvement by new highly accurate laboratory measurements in order to meet the ACCURATE requirements (Kirchengast and Schweitzer, 2011). They find such accurate spectroscopic measurements feasible with state-ofthe-art laser spectroscopy that targets single absorption lines so that spectroscopic uncertainties could be reduced to about $0.1 \%$ and line-center frequency uncertainties to within $10^{-8}$, thus meeting the requirements. We therefore did not include spectroscopic error modeling here, because we can assume that highly accurate spectroscopy will be available at launch of an ACCURATE-type mission and that the spectroscopic errors will then be minor to other errors accounted for here (e.g., thermal noise and residual errors from scintillations, as summarized below).

The HITRAN 2008 version used here includes a pressure shift parameter for the $\mathrm{C}^{18} \mathrm{OO}$ absorption line. This introduces an altitude dependency to the absorption frequencies. It would have been a special effort to implement the frequencies, which are currently implemented as scalars, as vectors within xEGOPS to take this dependency into account. We therefore disregarded the pressure shift parameter for the $\mathrm{C}^{18} \mathrm{OO}$ line in the present simulations, i.e., we set it to 0 . Syndergaard and Kirchengast (2015) confirmed that this is justified, because they fully included the pressure shift and found that the $\Delta k_{0}$ term in the Abel transform accurately compensates for its effects within the retrieval. Thus ignoring the pressure shift in the simulations here is a reasonable choice that does not limit the realistic character of the results obtained. For all other IR-laser channels for the GHG retrieval, pressure shift is included if HITRAN 2008 includes this information for the target absorption lines.

To further increase the realism of the simulations, an LIO scintillation model is included within the forward modeling sub-tool. The scintillation fluctuations are superimposed on the forward-modeled signals. This ensures that the influence of atmospheric turbulence, causing scintillations in LIO signals, is appropriately considered. The model is based on scintillation data from the GOMOS (Global Ozone Monitoring by Occultation of Stars) instrument (Sofieva et al., 2009) and was developed by Sofieva (2009) following the theoretical analyses of Horwath and Perlot (2008). The model is build on stratified altitude-dependent scintillations that saturate below about $20 \mathrm{~km}$. This is in agreement with GOMOS observations and experimental results by Gurvich et al. (1996). 

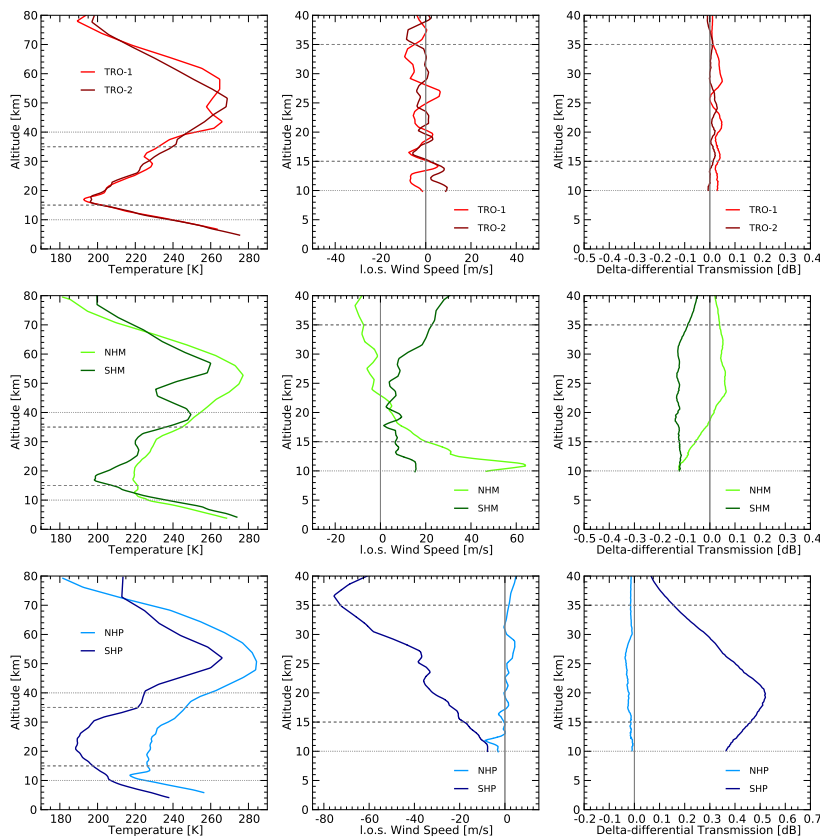

Figure 5. Temperature profiles (left), l.o.s. wind speed profiles (middle), and delta-differential transmission profiles (right) of the six selected atmospheric cases. The top row (red profiles) shows the TRO-1/2 cases, while the middle one (green profiles) shows the NHM and SHM cases and the bottom one (blue profiles) the NHP and SHP cases.

Individual signals of single frequency channels are strongly influenced by turbulence, as discussed by Schweitzer et al. (2011b). Although scintillations do fairly quickly decorrelate over frequency, the narrow spacing of absorption and reference channel of $<0.1 \%$ for the two wind channels (see Table 1) results in a very small influence of scintillations. For further details on scintillations see also Schweitzer et al. (2011b).

Following the forward modeling step, the influence of the observation system is modeled, employing the EGOPS/xEGOPS observation system modeling sub-tool. In this process, the observation system modeling superimposes observational errors on the forward modeled signals, which have included only the effects of atmospheric propagation. We add precise orbit determination errors, thermal noise errors, intensity drift, and clock errors in the same way as Proschek et al. (2011), who provided a more detailed description of the observation system modeling errors.

To complete the end-to-end simulations the retrieval subtool of the EGOPS/xEGOPS software is used. The thermodynamic state variables, including temperature, pressure, and humidity, are obtained from the LMO-based MW retrieval of EGOPS (Schweitzer et al., 2011a). These variables, in combination with the altitude level information, are the basis for the LIO-based IR retrieval of xEGOPS (Proschek et al., 2011). The new 1.o.s. wind speed retrieval, and the GHG re- trieval including correction of wind-induced Doppler shift, is embedded within the LIO retrieval algorithm as described in Sect. 2 above.

The influence of the 1.o.s. wind on the GHG retrieval is investigated at six geographical locations, representing a good diversity of atmospheric conditions: a warm and moist tropical atmosphere - tropical (TRO-1/2), a standard mid-latitude atmosphere - northern hemispheric mid-latitudes (NHM) and southern hemispheric mid-latitudes (SHM), and a cold and dry sub(ant)arctic winter atmosphere - northern hemispheric polar (NHP) and southern hemispheric polar (SHP).

In order to demonstrate the capability of the new l.o.s. wind retrieval and of the GHG algorithm under windy air conditions, the locations were chosen to include both highend wind velocities (especially represented by the polar cases) and quite variable vertical wind shear (especially represented by the tropical cases). The atmospheric thermodynamic and wind variables at the occultation event locations were extracted from ECMWF atmospheric fields, where we used an analysis field for providing "true" profiles to the forward modeling and a short-range forecast field for providing initial/background profiles to the retrieval.

The global distribution of the example occultation events for 15 July 2007, obtained with the near-polar satellite orbits (inclination $\approx 80^{\circ}$ ), is shown in Fig. 4 . The six selected occultation events are highlighted with colors according to their latitude. The ECMWF fields were used for the same day (15 July 2007, 12:00 UTC time layer).

Figure 5 presents the atmospheric conditions at the six occultation event locations. In the left column the temperature $(T)$ profiles for the six locations are shown. The middle column presents the "true" l.o.s. wind speed ( $\left.v_{\text {l.o.s. }}\right)$ profiles and the right column the corresponding delta-differential transmission $(\Delta \mathcal{T})$ profiles. The $T$ and $v_{\text {l.o.s. }}$ profiles are as used in the forward modeling, and the $\Delta \mathcal{T}$ profiles as obtained in the retrieval are the key input to the wind Abel transform (Eq. 2).

As seen in Fig. 5, the cases represent a good variety of different thermodynamic and windy air situations, including winds ranging from weak to strong wind speeds and from weak to strong vertical wind shears. The TRO- $1 / 2$ cases (top row, red profiles) show a sharp tropopause, rather weak but vertically highly variable winds, and as a result also weak $\Delta \mathcal{T}$ signals. The NHM and SHM cases (middle row, green profiles) exhibit more disturbed $T$ profiles, already somewhat stronger winds, and higher overall wind shear especially in the NHM case. The strongest l.o.s. wind speeds can be seen for the SHP case (bottom row, dark-blue profile), with values exceeding $70 \mathrm{~m} \mathrm{~s}^{-1}$ in the upper stratosphere, while a rather calm wind prevails in the NHP case. Therefore the SHP case also shows the strongest $\Delta \mathcal{T}$ signal. 

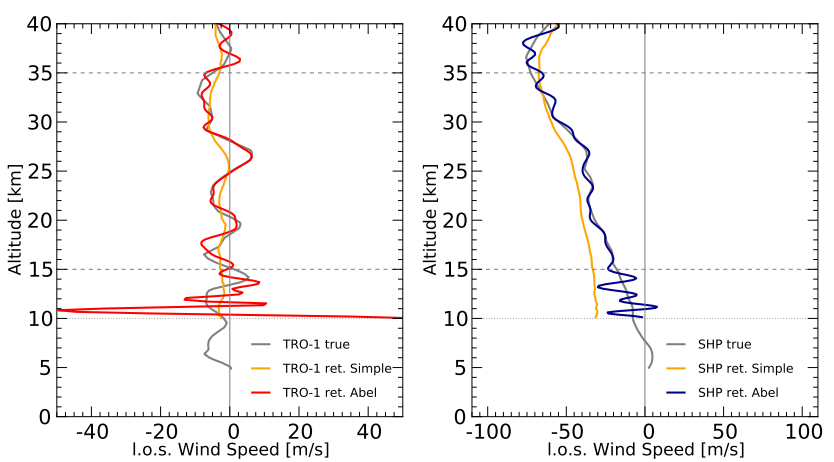

Figure 6. Abel transform and simple retrieval results for two representative cases. Left: simple retrieval (orange) and Abel transform retrieval (red) 1.o.s. wind speed for the TRO-1 case (red line). Right: simple retrieval (orange) and Abel transform retrieval (blue) results for the SHP case. The gray profile is, in both panels, the "true" profile used in the forward modeling of the events.

\section{Demonstration results}

Here we present results of the LMIO end-to-end simulations under windy air conditions according to the setup above in order to demonstrate the performance of the advanced retrieval. We start with a comparison of the new Abel transform-based wind retrieval with the previously introduced simple wind retrieval (Schweitzer, 2010; Kirchengast and Schweitzer, 2011).

Figure 6 shows the comparison of the new and the simple 1.o.s. wind retrieval for the TRO-1 and SHP example cases, which are neatly representing quite different conditions. The "true" l.o.s. wind speed profile is shown for reference. The SHP case shows high 1.o.s. wind speeds up to $75 \mathrm{~m} \mathrm{~s}^{-1}$, while the TRO-1 case shows comparatively small l.o.s. wind speeds but with higher wind shears. The new wind retrieval results are in very good agreement with the "true wind" over about 15 to $35 \mathrm{~km}$, both for weak and very strong wind speeds, while the simple wind retrieval results show major deviations and overly strict vertical smoothing at all altitude levels. This confirms the expectations of Schweitzer (2010) and Kirchengast and Schweitzer (2011) who argued that the formulation of an Abel transform-type retrieval algorithm, as then formulated by Syndergaard and Kirchengast (2013, 2015), should markedly improve the retrieval performance compared to the simple retrieval approach.

Figure 7 presents the 1.o.s. wind speed error in the form of a small-ensemble statistical result from all six cases. This result very clearly shows the superiority of the new wind retrieval (left panel) over the simple wind retrieval (right panel) both in avoiding biased retrieval and in reducing the r.m.s. error. The Abel transform-based retrieval is capable of achieving the scientific target requirements of $2 \mathrm{~m} \mathrm{~s}^{-1}$ r.m.s. error over most of the target domain from 15 to $35 \mathrm{~km}$ and retrieves within 5 to $40 \mathrm{~km}$. Below about $15 \mathrm{~km}$ the retrieval quality
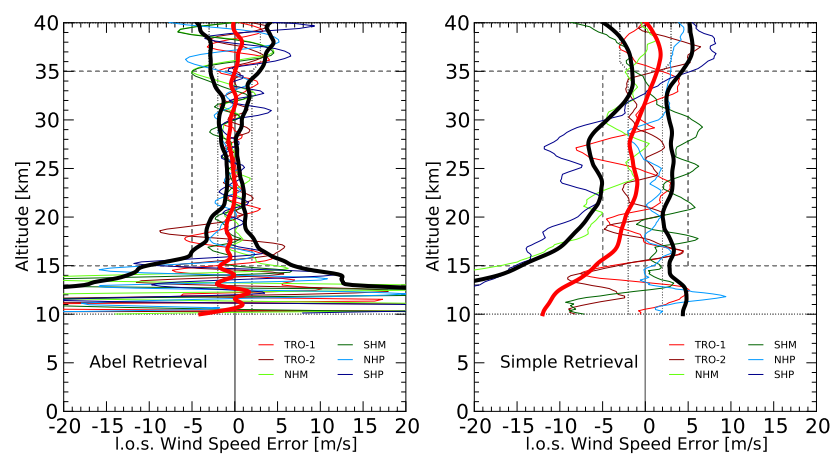

Figure 7. Statistical 1.o.s. wind speed errors for Abel transform retrieval (left) and simple retrieval (right) for the six example cases including TRO-1/2 (light red, red), NHM (light green), SHM (green), NHP (light blue), and SHP (blue). The heavy red lines show the estimated mean error profile, while the heavy black lines are the estimated SDs depicted as plus/minus envelope about the mean (both the mean and the SD are estimated at each vertical level using a $\pm 2 \mathrm{~km}$ averaging window to smooth out individual error oscillations due to the small ensemble). Target/threshold requirements for the errors are shown as dotted/dashed vertical lines; analogously, target altitude ranges are depicted as horizontal lines.

rapidly degrades due to the decreasing signal-to-noise ratio for the delta-differential transmission signals.

Building on the favorable performance of the Abel transform-based wind retrieval, Fig. 8 demonstrates the influences of the correction of a wind-induced Doppler shift on the GHG retrieval, again by using the two representative TRO-1 (left) and SHP (right) example cases. The GHG example species are $\mathrm{CO}_{2}$ (top) and $\mathrm{H}_{2} \mathrm{O}$ (bottom), both representing quite different LIO trace species retrieval challenges as seen by the studies of Proschek et al. (2011) and Proschek et al. (2014). VMR errors are shown and the retrieval results without Doppler shift correction (i.e., ignoring the windy air and assuming zero wind) are shown as gray profiles for reference. Overall we see that the VMR retrieval errors with the Doppler shift correction, and in case of sufficiently weak wind also without, basically appear to stay in an r.m.s. sense within the scientific target requirements of $2 \%$ for $\mathrm{CO}_{2}$ and $4 \%$ for $\mathrm{H}_{2} \mathrm{O}$.

Looking specifically at the TRO- 1 case, it is clear that the Doppler shift correction shows only a very minor influence (the red profiles essentially shadow the gray profiles), since the 1.o.s. wind speed at this tropical location is rather low (within $\pm 10 \mathrm{~m} \mathrm{~s}^{-1}$; see Fig. 5). This confirms the initial simple estimates of Schweitzer et al. (2011a) that wind speeds not exceeding $10 \mathrm{~m} \mathrm{~s}^{-1}$, or when the uncertainty of wind profiles used for the correction would not exceed this level, are not critical to the accuracy of the GHG retrievals. At the SHP location, with much higher 1.o.s. wind speeds up to $\approx 75 \mathrm{~m} \mathrm{~s}^{-1}$ in the upper stratosphere (see Fig. 5), the influence of the correction is easily visible, however. It provides for a clear improvement in this case, eliminating biased 

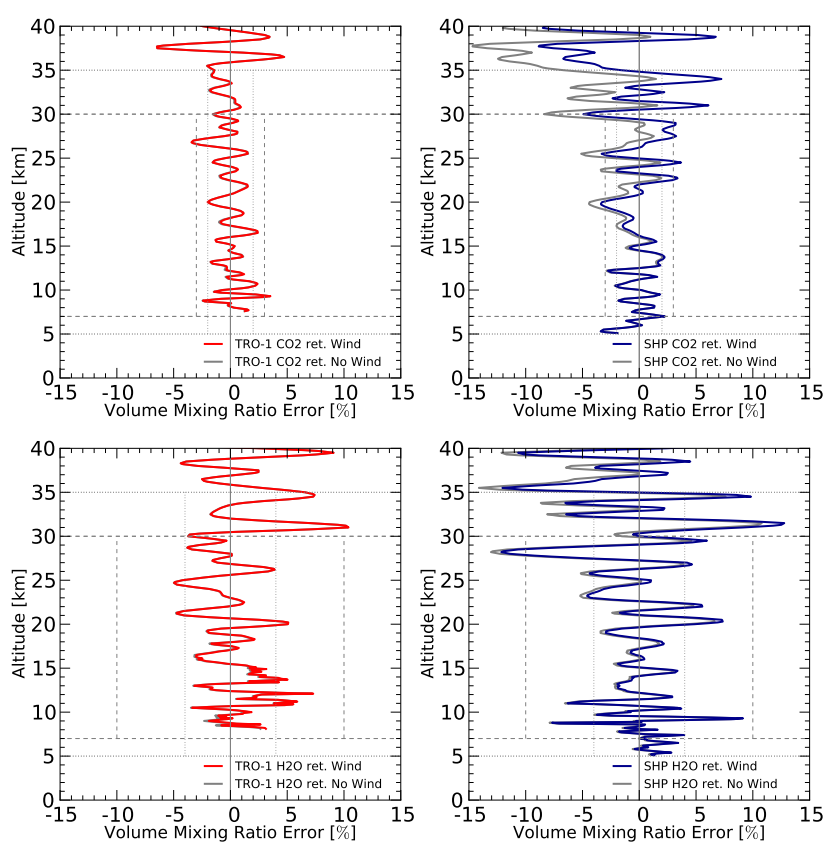

Figure 8. GHG volume mixing ratio errors with and without wind correction. The left column shows VMR results for $\mathrm{CO}_{2}$ (top) and $\mathrm{H}_{2} \mathrm{O}$ (bottom) for the TRO-1 case, and the right column is the same for the SHP case. The results without wind correction are shown as gray profiles while the results including the Doppler shift correction are shown in red (TRO-1 case) and blue (SHP case).

retrieval over the stratosphere. Here in particular the $\mathrm{CO}_{2}$ retrieval is more sensitive than the $\mathrm{H}_{2} \mathrm{O}$ retrieval due to somewhat different line shapes of the corresponding absorption lines.

One general conclusion here is that ECMWF short-range forecast wind fields not only aid the GHG retrieval with Doppler shift correction but also generally do a reasonable job in the troposphere, given that their wind uncertainties are typically smaller than $10 \mathrm{~m} \mathrm{~s}^{-1}$. Above $15 \mathrm{~km}$ the retrieved wind profiles are clearly preferable, since the uncertainties of the ECMWF analyses and short-range forecasts increase into the stratosphere due to the more sparse observations available for data assimilation at these altitudes. For climate monitoring purposes a self-consistent solution with joint wind and GHG retrieval from LMIO data is nevertheless preferable.

Figure 9 shows the VMR retrieval errors, including Doppler shift correction under the given windy air conditions, for the GHG species $\mathrm{CO}_{2}$ (upper left), $\mathrm{CH}_{4}$ (upper right), $\mathrm{H}_{2} \mathrm{O}$ (lower left), and $\mathrm{O}_{3}$ (lower right) in the form of a small-ensemble statistical result from all six selected occultation events. This serves to illustrate the overall performance achievable for different key species. The results for all species are essentially unbiased, which is very encouraging for LMIO implementation, since it is one core promise to qualify as a climate benchmark data set.
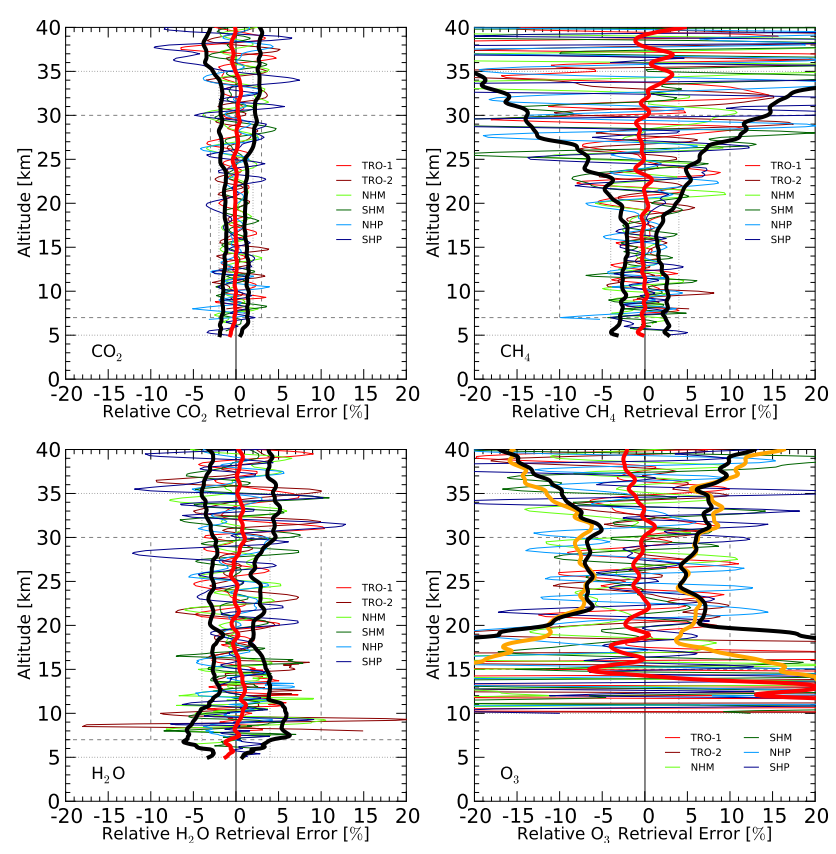

Figure 9. VMR retrieval error results for the six example cases TRO-1/2 (light red, red), NHM (light green), SHM (green), NHP (light blue), and SHP (blue) for retrievals of the GHGs $\mathrm{CO}_{2}$ (upper left), $\mathrm{CH}_{4}$ (upper right), $\mathrm{H}_{2} \mathrm{O}$ (lower left), and $\mathrm{O}_{3}$ (lower right), including the correction of wind-induced Doppler shift. The heavy red lines show the estimated mean error profile, and the heavy black lines are the estimated SDs depicted as plus/minus envelope about the mean (both the mean and the SD are estimated at each vertical level using a $\pm 2 \mathrm{~km}$ averaging window to smooth out individual error oscillations given the small ensemble). For $\mathrm{O}_{3}$ (lower right), the additional heavy orange lines mark the SD when computed from the four extratropical rather than all six cases, i.e., excluding the lowsignal-to-noise-ratio cases TRO-1/2. Target/threshold requirements for the errors are shown as dotted/dashed vertical lines; analogously, target altitude ranges are the horizontal lines.

The $\mathrm{CO}_{2}$ and $\mathrm{H}_{2} \mathrm{O}$ stay within scientific target requirements over most of the vertical domain, as already indicated in the single-profile results of Fig. 8. The $\mathrm{CH}_{4}$ results stay within requirements up to about $25 \mathrm{~km}$, above which the $\mathrm{CH}_{4}$ VMR becomes small and the signal-to-noise ratio degrades primarily due to residual scintillation noise, which governs the noise budget at these altitudes.

Finally, the $\mathrm{O}_{3}$ results are within the requirements over the stratospheric ozone layer down to about 20 to $15 \mathrm{~km}$, where the downward increase of errors strongly depends on whether tropical cases with very low (tropospheric) ozone concentrations up to about $18 \mathrm{~km}$ are involved or not, since the extratropical air is richer in ozone lower down. Compared to the earlier $\mathrm{O}_{3}$ retrieval performance results of Proschek et al. (2011), these $\mathrm{O}_{3}$ results also reflect that the new $\mathrm{O}_{3}$ channel selections (near $4670 \mathrm{~cm}^{-1}$ rather than near $4030 \mathrm{~cm}^{-1}$ as originally selected by Kirchengast and Schweitzer (2011); see Table 1) exhibit somewhat less absorber sensitivity (but 
are technically much more convenient to implement together with all other channels).

\section{Summary and conclusions}

We introduced an advanced l.o.s. wind speed retrieval, based on a new wind Abel transform (Syndergaard and Kirchengast, 2013, 2015), into the existing LMIO algorithm described by Proschek et al. (2011). Making use of this new wind retrieval we also integrated a correction for windinduced Doppler shift into the GHG retrieval algorithm, which might otherwise lead to slightly biased retrievals under windy air conditions.

We described the new l.o.s. wind speed retrieval and the Doppler shift correction in the GHG retrieval and evaluated the performance of the advanced LMIO algorithm based on realistic end-to-end simulations for six representative occultation events spanning a variety of atmospheric conditions from tropical to high-latitude winter. The retrieval was performed for the thermodynamic state variables temperature, pressure, and humidity from the microwave channels and for the 1.o.s. wind speed from the two IR-laser wind channels at the highly symmetric $\mathrm{C}^{18} \mathrm{OO}$ absorption line. The GHG retrievals from IR-laser channels were performed for the representative species $\mathrm{CO}_{2}, \mathrm{CH}_{4}, \mathrm{H}_{2} \mathrm{O}$, and $\mathrm{O}_{3}$.

For assessing the performance of the new Abel transform wind retrieval it was compared to the initial simple wind retrieval approach of Schweitzer (2010) and Kirchengast and Schweitzer (2011). This showed significant benefit and improvement from the new Abel transform retrieval, since it can account for the spherical symmetry of the problem while the simple wind retrieval strongly over-smoothes vertical wind variations.

The results for the six example occultation events showed the reliability of the Abel transform retrieval for providing 1.o.s. wind speeds in an essentially unbiased manner and generally with errors within the target requirement range of $2 \mathrm{~m} \mathrm{~s}^{-1}$ throughout the lower stratosphere over about 15 to $35 \mathrm{~km}$. Below $15 \mathrm{~km}$ the retrieval errors increase rapidly due to the decreasing signal-to-noise ratio of the deltadifferential transmission observable. The simple wind retrieval is not capable of reliably deriving realistic l.o.s. winds within scientific observation requirements. This underscores the importance of the new Abel transform in windy air, including under strong winds conditions, for enabling accurate wind profiling based on IR-laser occultation data.

The correction of the wind-induced Doppler shift influence in the GHG retrieval shows significant benefit for the GHG retrieval results, in particular in the case of strong winds. Weak 1.o.s. winds with speeds not exceeding $10 \mathrm{~m} \mathrm{~s}^{-1}$ have no appreciable influence on the GHG results, as anticipated by Schweitzer et al. (2011b) based on preliminary estimates. This is favorable since it implies that winds need not be more accurate than about $10 \mathrm{~m} \mathrm{~s}^{-1}$ for the purpose of supporting GHG retrieval. This also justifies the use of co- located winds from ECMWF analysis or short-range forecast fields in the troposphere below $15 \mathrm{~km}$, where the retrieved winds are of lower quality.

The GHG retrieval results in windy air were found promising, since all retrieved species $\left(\mathrm{CO}_{2}, \mathrm{CH}_{4}, \mathrm{H}_{2} \mathrm{O}\right.$, and $\left.\mathrm{O}_{3}\right)$ were found essentially unbiased and generally within the scientific target requirement ranges of 2 to $4 \%$ over the upper troposphere and lower stratosphere. $\mathrm{CO}_{2}$ and $\mathrm{H}_{2} \mathrm{O}$ are of very coherent quality throughout this range. $\mathrm{CH}_{4}$ errors increase above about $25 \mathrm{~km}$ and $\mathrm{O}_{3}$ errors below about 20 to $15 \mathrm{~km}$, both due to the decreasing signal-to-noise ratio in these upper/lower domains, where the noise is dominated by residual scintillation noise as also found by Proschek et al. (2014).

Overall the new Abel transform wind retrieval is found to enable accurate stratospheric wind profiling based on IRlaser occultation data in windy air, including under strong wind conditions and variable vertical wind shears. The GHG retrieval, including correction for the l.o.s. wind-induced Doppler shift, also shows robust and accurate retrieval results under all windy air conditions and therefore enables benchmark-quality greenhouse gas profiling in windy air. These results are encouraging for further development and implementation of an LMIO mission.

Acknowledgements. We thank S. Syndergaard (DMI, DK) for valuable discussions on the application of the wind Abel transform and for the excellent collaboration in its development. EGOPS was developed by an international consortium led by UniGraz (AT) and involving partners at Danish Meteorological Institute (DK), Obukhov Institute of Atmospheric Physics (RU), Chalmers University of Technology (SE), University of Bremen (DE), Met. Office (UK), Terma Elektronik A/S (DK), and RUAG Space $\mathrm{GmbH}$ (AT). xEGOPS was developed by UniGraz (AT), with contributions by E. Martini (CNIT, IT) and V. Sofieva (FMI, FI) to scintillation modeling and by $\mathrm{C}$. Emde (University of Munich, DE) to cloud extinction modeling. Funds for the EGOPS/xEGOPS development were provided by ESA/ESTEC (NL), FWF and FFG-ALR (AT), and EUMETSAT/HQ (DE). Access to atmospheric analysis fields was provided by the ECMWF, access to RFM and FASCODE by A. Dudhia (University of Oxford, UK) via www.atm.ox.ac.uk/RFM, and access to HITRAN by L. Rothman (Harvard University, USA) via www.cfa.harvard.edu/hitran. This work was funded by ESA under the AEXPWIND project (ESA/ESTEC contract no. 4000105679/12/NL/CBi).

Edited by: J. Y. Liu

\section{References}

Andrews, L. C. and Philips, R. L.: Laser Beam Propagation Through Random Media, 2nd edn., SPIE Press, Bellingham, Washington, USA, 2005.

Dudhia, A.: Reference Forward Model RFM: Inst. of Atmos., Oceanic and Planet. Phys., Univ. of Oxford, Oxford, UK, available at: http://www.atm.ox.ac.uk/RFM/ (last access: 06 July 2015), 2008. 
Edwards, D. P.: High level algorithm definition document, Tech. Rep. ESA/ESTEC PO-TN-OXF-GS-0004, Contract No. 11886/96/NL/GS, Inst. of Atmos., Oceanic and Planet. Phys., Univ. of Oxford, Oxford, UK, 1996.

FASCODE: cited 2008 on RFM website - FASCODE model atmospheres: Inst. of Atmos., Oceanic and Planet. Phys., Univ. of Oxford, Oxford, UK, available at: http://www.atm.ox.ac.uk/RFM/ atm (last access: 06 July 2015), 2008.

Fritzer, J. M., Kirchengast, G., and Pock, M.: End-to-End Generic Occultation Performance Simulation and Processing System version 5.5 (EGOPS 5.5) Software User Manual, Tech. Rep. ESAESTEC WEGC-EGOPS-2009-TR01, Wegener Center and Inst. for Geophys., Astrophys., and Meteorol., Univ. of Graz, Graz, Austria, 2009.

Fritzer, J. M., Kirchengast, G., Pock, M., and Proschek, V.: Endto-End Generic Occultation Performance Simulation and Processing System version 5.5 (EGOPS 5.5 and xEGOPS) Software User Manual, Tech. Rep. ESA-ESTEC WEGC-EGOPS-2010TR01, Wegener Center and Inst. for Geophys., Astrophys., and Meteorol., Univ. of Graz, Graz, Austria, 2010.

Gurvich, A. S., Kan, V., and Fedorova, O. V.: Refraction angle fluctuations in the atmosphere from space observations of stellar scintillations, Atmospheric and Oceanic Physics, 31, 742-749, englisch version, 1996.

Gurvich, A. S., Gorbunov, M. E., Fedorova, O. V., Kirchengast, G., Proschek, V., González Abad, G., and Tereszchuk, K. A.: Spatiotemporal structure of a laser beam over $144 \mathrm{~km}$ in a Canary Islands experiment, Appl. Optics, 51, 7374-7383, doi:10.1364/AO.51.007374, 2012.

Harrison, J. J., Bernath, P. F., and Kirchengast, G.: Spectroscopic requirements for ACCURATE, a microwave and infrared-laser occultation satellite mission, J. Quant. Spectrosc. Ra., 112, 23472354, 2011.

Horwath, J. and Perlot, N.: Atmospheric impacts on ILO signals: Scintillation, Tech. Rep. for ESA-ESTEC Technical Note 4 - Part of the TR-IRPERF Report, DLR Oberpfaffenhofen, Oberpfaffenhofen, Germany, 2008.

Kirchengast, G. and Schweitzer, S.: Climate benchmark profiling of greenhouse gases and thermodynamic structure and wind from space, Geophys. Res. Lett., 38, L13701, doi:10.1029/2011GL047617, 2011.

Kirchengast, G., Bernath, P. F., Buehler, S., Durry, G., Facheris, L., Gerbig, C., Haimberger, L., Harris, J., Hauchecorne, A., Kurölä, E., Larsen, G. B., Sausen, R., Anthes, R. A., Gorbunov, M. E., Kursinski, E. R., Leroy, S. S., Trenberth, K., Randel, B., Gille, J., and Tsuda, T.: ACCURATE - climate benchmark profiling of greeenhouse gases and thermodynamic variables and wind from space (ESA Earth Explorer Opportunity Mission EE-8 proposal), Sci. Rep. No. 36, document wcv-scirep-no36-gkirchengastetal-jul2010.pdf, Wegener Center Verlag, Graz, Austria, available at: http://www.wegcenter.at/wcv (last access: 06 July 2015), 2010.

Kursinski, E. R., Hajj, G. A., Schofield, J. T., Linfield, R. P., and Hardy, K. R.: Observing Earth's atmosphere with radio occultation measurements using the global positioning system, J. Geophys. Res.-Atmos., 102, 23429-23465, 1997.

Kursinski, E. R., Hajj, G. A., Leroy, S. S., and Herman, B.: The GPS radio occultation technique, Terr. Atmos. Ocean. Sci., 11, 53-114, 2000.
Kursinski, E. R., Syndergaard, S., Flittner, D., Feng, D., Hajj, G., Herman, B., Ward, D., and Yunck, T.: A microwave occultation observing system optimized to characterize atmospheric water, temperature and geopotential via absorption, J. Atmos. Ocean. Tech., 19, 1897-1914, doi:10.1175/15200426(2002)019<1897:AMOOSO>2.0.CO;2, 2002.

Liou, K. N.: An Introduction to Atmospheric Radiation, Academic Press, San Diego, California, USA, 2nd edn., 2002.

Proschek, V., Kirchengast, G., and Schweitzer, S.: Greenhouse gas profiling by infrared-laser and microwave occultation: retrieval algorithm and demonstration results from end-to-end simulations, Atmos. Meas. Tech., 4, 2035-2058, doi:10.5194/amt-42035-2011, 2011.

Proschek, V., Kirchengast, G., Emde, C., and Schweitzer, S.: Greenhouse gas profiling by infrared-laser and microwave occultation in cloudy air: results from end-to-end simulations, J. Geophys. Res. Atmos., 119, 12372-12390, doi:10.1002/2014JD021938, 2014.

Rothman, L. S., Rinsland, C. P., Goldman, A., Massie, S. T., Edwards, D. P., Flaud, J.-M., Perrin, A., Camy-Peyret, C., Dana, V., Mandin, J.-Y., Schroeder, J., McCann, A., Gamache, R. R., Wattson, R. B., Yoshino, K., Cahnce, K. V., Jucks, K. W., Brown, L. R., Nemtchinov, V., and Varanasi, P.: The HITRAN molecular spectrsoscopic database and HAWKS (Hitran Atmospheric WorKStation): 1996 edition, J. Quant. Spectrosc. Ra., 60, 665-710, 1998.

Rothman, L. S., Gordon, I. E., Barbe, A., Benner, D. C., Bernath, P. F., Birk, M., Boudon, V., Brown, L. R., Campargue, A., Champion, J.-P., Chance, K., Coudert, L. H., Dana, V., Devi, V. M., Fally, S., Flaud, J.-M., Gamache, R. R., Goldman, A., Jacquemart, D., Kleiner, I., Lacome, N., Lafferty, W. J., Mandin, J.-Y., Massie, S. T., Mikhailenko, S. N., Miller, C. E., Moazzen-Ahmadi, N., Naumenko, O. V., Nikitin, A. V., Orphal, J., Perevalov, V. I., Perrin, A., Predoi-Cross, A., Rinsland, C. P., Rotger, M., Šimečková, M., Smith, M. A. H. Sung, K., Tashkun, S. A., Tennyson, J., Toth, R. A., Vandaele, A. C., and Vander Auwera, J.: The HITRAN 2008 molecular spectroscopic database, J. Quant. Spectrosc. Ra., 110, 533572, doi:10.1016/j.jqsrt.2009.02.013, 2009.

Rothman, L. S., Gordon, I. E., Babikov, Y., Barbe, A., Benner, D. C., Bernath, P. F., Birk, M., Bizzocchi, L., Boudon, V., Brown, L. R., Campargue, A., Chance, K., Cohen, E. A., Coudert, L. H., Devi, V. M., Drouin, B. J., Fayt, A., Flaud, J.M., Gamache, R. R., Harrison, J. J., Hartmann, J.-M., Hill, C., Hodges, J. T., Jacquemart, D., Jolly, A., Lamouroux, J., Roy, R. J. L., Li, G., Long, D. A., Lyulin, O. M., Mackie, C. J., Massie, S. T., Mikhailenko, S., Mueller, H. S. P., Naumenko, O. V., Nikitin, A. V., Orphal, J., Perevalov, V., Perrin, A., Polovtseva, E. R., Richard, C., Smith, M. A. H., Starikova, E., Sung, K., Tashkun, S., Tennyson, J., Toon, G. C., Tyuterev, V. G., and Wagner, G., The HITRAN2012 molecular spectroscopic database, Journal of Quantitative Spectroscopy and Radiative Transfer, 130, 4-50, 10.1016/j.jqsrt.2013.07.002, 2013

Salby, M. L.: Physics of the Atmosphere and Climate, Cambridge University Press, New York, 2012.

Schweitzer, S.: The ACCURATE concept and the infrared laser occultation technique: Mission design and assessment of retrieval performacne (Ph. D. thesis), Sci. Rep. No. 34, document wcvscirep-no34-sschweitzer-jun2010.pdf, Wegener Center Verlag, 
Graz, Austria, available at: http://www.wegcenter.at/wcv (last access: 06 July 2015), 2010.

Schweitzer, S., Kirchengast, G., and Proschek, V.: Atmospheric influences on infrared-laser signals used for occultation measurements between Low Earth Orbit satellites, Atmos. Meas. Tech., 4, 2273-2292, doi:10.5194/amt-4-2273-2011, $2011 \mathrm{a}$.

Schweitzer, S., Kirchengast, G., Schwärz, M., Fritzer, J. M., and Gorbunov, M. E.: Thermodynamic state retrieval from microwave occultation data and performance analysis based on end-to-end simulations, J. Geophys. Res. Atmos., 116, D10301, doi:10.1029/2010JD014850, 2011b.

Sofieva, V. F..: Atmospheric impacts on ILO signals: Assessment of infrared scintillations, Tech. Rep. for ESA-ESTEC Technical Note 3 - Part of the TR-IRPERF Report, Finnish Meteorological Institute, Helsinki, Finland, 2009.

Sofieva, V. F., Kan, V., Dalaudier, F., Kyrölä, E., Tamminen, J., Bertaux, J.-L., Hauchecorne, A., Fussen, D., and Vanhellemont, F.: Influence of scintillation on quality of ozone monitoring by GOMOS, Atmos. Chem. Phys., 9, 9197-9207, doi:10.5194/acp-9-9197-2009, 2009.
Syndergaard, S. and Kirchengast, G.: Formulation and analysis of an Abel transform for deriving line-of-sight wind profiles from LEO-LEO IR laser occulation, Tech. Rep. ESA-ESTEC-1/2013 (AEXPWIND TN3-Part1), Wegener Center, Univ. of Graz, Graz, Austria, 2013.

Syndergaard, S. and Kirchengast, G.: An Abel transform for deriving line-of-sight wind profiles from LEO-LEO infrared laser occultation measurements, J. Geophys. Res. Atmos., in review, 2015.

Ware, R., Exner, M., Feng, D., Gorbunov, M. E., Hardy, K., Herman, B. M., Kuo, W., Meehan, T., Melbourne, W., Rocken, C., Schreiner, W., Sokolovskiy, S., Solheim, F., Zou, X., Anthes, R., Businger, S., and Trenberth, K.: GPS sounding of the atmosphere from low Earth orbit: preliminary results, B. Am. Meteorol. Soc., 77, 19-40, 1996. 